\title{
Expression of neuronal nitric oxide synthase in the hippocampal formation in affective disorders
}

\author{
R.M.W. Oliveira ${ }^{1}$, F.S. Guimarães ${ }^{2}$ and J.F.W. Deakin ${ }^{3}$ \\ 1'Departamento de Farmácia e Farmacologia, Universidade Estadual de Maringá, Maringá, PR, Brasil \\ ${ }^{2}$ Faculdade de Medicina de Ribeirão Preto, Universidade de São Paulo, Ribeirão Preto, SP, Brasil \\ ${ }^{3}$ Neuroscience and Psychiatry Unit, The University of Manchester, Manchester, UK
}

Correspondence to: R.M.W. Oliveira, Departamento de Farmácia e Farmacologia, Universidade Estadual de Maringá, Avenida Colombo, 5790, 87020-900 Maringá, PR, Brasil

Fax: +55-44-3261-4999. E-mail: rmmwoliveira@uem.br

\begin{abstract}
Hippocampal output is increased in affective disorders and is mediated by increased glutamatergic input via $N$-methyl- $D$ aspartate (NMDA) receptor and moderated by antidepressant treatment. Activation of NMDA receptors by glutamate evokes the release of nitric oxide (NO) by the activation of neuronal nitric oxide synthase (nNOS). The human hippocampus contains a high density of NMDA receptors and nNOS-expressing neurons suggesting the existence of an NMDA-NO transduction pathway which can be involved in the pathogenesis of affective disorders. We tested the hypothesis that nNOS expression is increased in the human hippocampus from affectively ill patients. Immunocytochemistry was used to demonstrate nNOS-expressing neurons in sections obtained from the Stanley Consortium postmortem brain collection from patients with major depression (MD, $N=15)$, bipolar disorder (BD, $N=15)$, and schizophrenia $(N=15)$ and from controls $(N=15)$. nNOS-immunoreactive ( $n N O S-$ IR) and Nissl-stained neurons were counted in entorhinal cortex, hippocampal CA1, CA2, CA3, and CA4 subfields, and subiculum. The numbers of Nissl-stained neurons were very similar in different diagnostic groups and correlated significantly with the number of nNOS-IR neurons. Both the MD and the BD groups had greater number of nNOS-IR neurons/400 $\mu \mathrm{m}^{2}$ in CA1 (mean \pm SEM: $\mathrm{MD}=9.2 \pm 0.6$ and $\mathrm{BD}=8.4 \pm 0.6)$ and subiculum $(\mathrm{BD}=6.7 \pm 0.4)$ when compared to control group (6.6 \pm 0.5$)$ and this was significantly more marked in samples from the right hemisphere. These changes were specific to affective disorders since no changes were seen in the schizophrenic group $(6.7 \pm 0.8)$. The results support the current view of the NMDA-NO pathway as a target for the pathophysiology of affective disorders and antidepressant drug development.
\end{abstract}

Key words: Affective disorders; Major depression; Neuronal nitric oxide synthase; Immunohistochemistry; Hippocampus

Research supported by the Stanley Foundation, Muscatine, IA, USA, and CAPES. R.M.W. Oliveira was the recipient of a fellowship from CAPES. Publication supported by FAPESP.

Received September 10, 2007. Accepted March 4, 2008

\section{Introduction}

Affective disorders, consisting mainly of major depression (MD) and bipolar disorder (BD), are among the most severe diseases regarding disability-adjusted life years. Despite better diagnostic options and increasing awareness of $\mathrm{MD}$ and $\mathrm{BD}$, their pathophysiology remains elusive (1).

Although almost certainly not solely responsible for the myriad of symptoms observed in affective disorders, the highly plastic, stress-sensitive hippocampal formation may play a central role in affective disorders and in the action of antidepressant drugs (1). Despite variation in the measurement techniques and patient samples, structural magnetic resonance imaging studies have shown a significant reduction of the hippocampal volume in subjects with a history of affective disorder $(2,3)$ although some studies have reported negative findings (for a review, see Ref. 1). It appears that hippocampal atrophy is observed preferen- 
tially in older, recurrently depressed subjects or subjects who are refractory to antidepressant medications (4).

Experimentally, recurrent episodes of stress or elevated glucocorticoid levels, also characteristic of MD, are associated with damage to the hippocampus. A loss of hippocampal neurons is observed in monkeys submitted to prolonged psychosocial stress (5), whereas repeated restraint stress in rats causes hippocampal CA3 pyramidal neurons to undergo dendritic atrophy (6). These specific effects have been suggested to be mediated by activation of the hypothalamic-pituitary-adrenal axis acting in concert with excitatory amino acid input via the $N$-methyl- $D$-aspartate (NMDA) receptors (6)

In the brain, activation of the NMDA receptor by glutamate can release nitric oxide (NO) by the activation of calmodulin-calcium-dependent enzyme neuronal NO synthase (nNOS) (7). nNOS catalyzes the oxidation of Larginine resulting in the formation of L-citrulline and $\mathrm{NO}$, which is a diffusible, free-radical messenger involved in cell-cell communication and in numerous physiologic and neuropsychiatric conditions $(8,9)$.

The human hippocampus contains a high density of NMDA receptors (10) and nNOS-expressing cells $(11,12)$, suggesting the existence of an NMDA-NO transduction pathway. A crucial involvement of the NMDA-NO signaling pathway in the pathophysiology of MD and in the mechanism of action of antidepressant drugs has been suggested. For example, NMDA receptor antagonists or NOS inhibitors exhibit antidepressant-like properties in animal screening procedures (13-15). In addition, chronic treatment with an NOS inhibitor also down-regulates $ß$-adrenergic receptors in the frontal cortex of mice with a magnitude comparable to imipramine (16). Clinically, ketamine, an NMDA receptor antagonist, exhibits antidepressant activity in humans (17) as does methylene blue, an NOS inhibitor that was found to be therapeutically effective in severe MD (18). Finally, NO production may increase MD since the patients show elevate plasma nitrate levels (19).

Studies in animals and humans have shown that the hippocampus may be also an important site of action of antidepressant drugs. Systemic and intra-hippocampal administration of antidepressants prevent the anxiety incubation effect of restraint stress and the development of learned helplessness (15). Compellingly, in a PET study of MD, Mayberg et al. (20) reported that clinical recovery on fluoxetine was associated with a reduction in hippocampal metabolism. This suggests that antidepressants decrease hippocampal glutamatergic function and consequently NO function since regional cortical metabolism mainly accounts for glutamatergic neurotransmission (21). In this way, it has been demonstrated that inhibition of the NMDA-NO pathway appears to be one of the common actions of antidepressants. Despite remarkable structural diversity, three main kinds of antidepressants (desipramine, fluoxetine and moclobemide) not only antagonized the NMDAinduced lesion in PC12 cells, but also inhibited the function of the NMDA-NO signal pathway (22). These findings are consistent with the hypothesis first proposed by Paul and Skolnick (14) that clinically used antidepressants may share a common ability to block or down-regulate NMDA glutamate receptor function.

In 1991, Deakin and Graeff (23) proposed that impairment of hippocampal serotonin function weakens behavioral resilience and results in MD. It has been long proposed that impaired serotonin function in MD may be mediated by stress-induced hypercortisolemia acting on the high concentration of glucocorticoid receptors in the hippocampus (1). Studies by Joca and Guimarães (15) and others (6) have suggested that stress-induced changes in hippocampal glutamate mechanisms may precede changes in serotonin function.

Recently, it has been suggested that hippocampal NO under non-stressed conditions may be under tonic inhibition by serotonergic neurons since an increased NOS activity in the context of long-term $5-\mathrm{HT}_{2}$ receptor inhibition by ritanserin was observed in rats submitted to forced swimming stress (24). Furthermore, this control might be lost following states of serotonergic depletion, manifesting as learned helplessness in animals and as depression in humans.

Taken together, these findings suggest that activation of the NMDA-NO pathway in the hippocampus can be involved in the pathogenesis of affective disorders, possibly triggered by glucocorticoid-mediated effects of stress and mediated by impaired serotonin function. To investigate this hypothesis in humans, we have tested the prediction that expression of nNOS is increased in the hippocampus obtained post mortem from patients suffering from $\mathrm{MD}$ and $\mathrm{BD}$.

\section{Material and Methods}

\section{Tissue samples}

Sections of the hippocampus were obtained from the Stanley Medical Research Institute Neuropathology Consortium (25). The material was obtained from 15 controls, 15 schizophrenic (SCZ), $15 \mathrm{MD}$, and $15 \mathrm{BD}$ cases. Clinical and demographical data are shown in Table 1. The psychiatric diagnosis was based on retrospective case note review applying the criteria of the Diagnostic and Statistical Manual of Mental Disorders, 4th edition, 1994. Normal controls had no history of neurological or psychiatric illness and neuropathological examination excluded Alzhei- 
mer's disease or other cerebral pathology. The blocks were fixed in $10 \%$ phosphate-buffered formalin, $\mathrm{pH} 7.0$, for 1 to 31 months (average 8 months) and then embedded in paraffin. Ten-micrometer sections were cut, mounted onto gelatin-coated slides and stored at room temperature until processed for nNOS immunohistochemistry.

\section{Immunohistochemistry}

The sections were initially dewaxed in two changes of xylene (20 min each), rehydrated through graded alcohols (100, 95, 80, and 70\% ethanol, 5 min each), washed in distilled water and pre-treated with $3 \% \mathrm{H}_{2} \mathrm{O}_{2}$ in $50 \%$ methanol (30 $\mathrm{min}$ ) to decrease endogenous peroxidase. After repeated washings ( 3 X $15 \mathrm{~min}$ ) in $0.1 \mathrm{M}$ phosphate-buffered saline (PBS), $\mathrm{pH} 7.4$, the sections were incubated with $5 \%$ normal goat serum in $0.1 \mathrm{M}$ PBS containing $0.3 \%$ Triton X-100 (PBS-T) for $1 \mathrm{~h}$, to block nonspecific binding sites. After rinsing in $0.1 \mathrm{M}$ PBS-T, the sections were incubated with the primary antibody (1:25, mouse antinNOS, clone 16, Transduction Laboratories, San Diego, CA, USA) for $40 \mathrm{~h}$ at $4^{\circ} \mathrm{C}$. The primary antibody was washed off with PBS-T ( 3 X $15 \mathrm{~min}$ ) and the sections were then incubated with biotinylated goat anti-mouse immunoglobulin G (1:100, PharmiGen, San Diego, CA, USA) for 2 $h$. They were then rinsed in PBS-T before 1-h incubation with the avidin-biotin-peroxidase complex (1:500, Vector Laboratories, Burlingame, CA, USA). Peroxidase activity was developed using 3'-3' diaminobenzidine tetrahydrochloride (Sigma, USA) containing $0.05 \% \mathrm{H}_{2} \mathrm{O}_{2}$. Finally, the sections were dehydrated through a series of alcohols (70, 80,95 , and $100 \%$ ), cleared in xylene and cover-slipped.

All reactions were carried out at the same time. Control experiments were performed by omitting the primary antibody from the staining protocol. Adjacent sections were stained with Cresyl violet (Nissl preparation) to help identify the location of the areas of interest.

\section{Quantification}

Microscope images were captured using a video camera (Sony Hyper HAD) linked to a Leica DMRB (Nussloch, Germany) microscope. nNOS-immunoreactivity (nNOSIR) was considered to be present when the cell showed a clear unstained nucleus with granular immunoreactivity in the cytoplasm. A two-dimensional counting method was used to estimate immunopositive cell number within 400$\mu \mathrm{m}^{2}$ counting frames. Sections were viewed at $200 \mathrm{X}$ magnification and images were captured using the Bioquant (Nova Prime, Nashville, TN, USA) image analysis system. Cells were counted in three to five fields $\left(400 \mu \mathrm{m}^{2}\right)$ distributed across each area of interest per section, in three of the four sections available per subject. The fourth section was used for Nissl staining. All, nNOS-IR or Nissl-stained neurons lying within counting frames were counted, excluding those that crossed either the top or left side of the frame (26). The images were carefully checked to ensure that all neurons were recognized, that contiguous cells were split and that the classification was corrected with the exclusion of vessels and glia. These criteria were in accordance with previously published studies $(27,28)$. Cell counts were carried out in a blind manner, without access to any information about diagnosis by RMWO and the statistical analysis was carried out by JFWD who had no involvement in counting.

The following regions were selected for analysis with reference to the maps published by Paxinos and Mai (29): CA4, CA3, CA2, and CA1 pyramidal layers of the hippocampus, pyramidal layer III of the subiculum and layers II and III of the entorhinal cortex. Laminar boundaries were confirmed with the aid of an encoder measuring the position from the pial surface to the borders of each layer of adjacent Nissl-stained sections which in all cases closely overlay the nNOS sections. In the dentate gyrus, cells were too densely packed to be counted. It was not possible to identify all sub-regions in every subject because of variation in the anteroposterior level of sectioning. However, there were no systematic group differences in sectioning level.

\section{Statistical analysis}

To identify potential confounders to be included as covariates, analysis of variance (ANOVA) was used to determine group differences in age, $\mathrm{pH}$, interval between death and fixation (postmortem interval, $\mathrm{PMI}$ ), and duration of storage in formalin. In addition, correlations were computed between cell counts and the potential confounders. The average number of nNOS-IR and Nissl-stained neurons in each region was obtained, and the mean for each of the four groups including controls, SCZ, MD, and BD was further calculated. Data were analyzed using ANOVA with factors for diagnosis (4 levels) and side (2 levels). Side was a between-subject factor because leftand right-sided samples came from different brains since in the Stanley collection one hemisphere is fixed and the other frozen. In the SCZ group, there were insufficient observations ( $N=3-4$ per region) in the right hippocampus to permit reliable identification of lateralized abnormalities (i.e., diagnosis by side interactions). Therefore, to determine whether diagnosis by side interactions in the main analysis was due to lateralized changes in the MD and $B D$ groups, the main ANOVA was repeated including only MD, $\mathrm{BD}$ and controls. The origin of diagnosis by side interactions was determined by unilateral ANOVA with planned 
contrasts. Finally, analysis of covariance was carried out for the affective and control groups with Nissl-stained neuron numbers as a covariate.

To test the hypothesis that nNOS-IR neurons would be reduced in subjects taking antidepressants and lithium, counts from the BD and MD groups were combined and compared in the treatment groups by the $t$-test. To test the hypothesis that nNOS counts would be reduced in subjects with a long duration of illness, Spearman correlations between counts and duration of illness were calculated for the combined BD plus MD group.

\section{Results}

\section{Confounders}

Age and gender did not differ between the diagnostic and control groups and did not correlate with nNOS counts. Patients tended to have longer PMls and although the main effect of diagnosis was not statistically significant, planned contrasts showed that greater PMls were significant in the SCZ group $(P=0.04)$ and borderline in the BD group $(P=0.07 ;$ Table 1$)$. However, $P M I$ did not correlate with nNOS counts and was not retained as a covariate. Brains that had been exposed to formalin for substantially longer periods than brains from controls (main effect of diagnosis: $F=3.55$, d.f. $=3.56, P=0.02$ ) and 9 of the 15 controls had shorter fixation times than any of the SCZ group and most of the other groups. However, time in formalin did not correlate with nNOS counts and was not included as a covariate. As an additional check, ANOVA was repeated excluding controls since the clinical groups did not differ in fixation time.

\section{Distribution of nNOS-IR neurons in the hippocampal formation}

Neurons expressing nNOS-IR were distributed in all fields of the hippocampus (CA4, CA3, CA2, and CA1), dentate gyrus, subiculum, and entorhinal cortex (Figure 1).

Table 1. Clinical and demographic data of patients and controls from Stanley Consortium postmortem brain collection.

\begin{tabular}{lcccc}
\hline & \multicolumn{4}{c}{ Psychiatric diagnosis } \\
\cline { 2 - 5 } & Negative & SCZ & MD & BD \\
\hline Age (years) & $49.0 \pm 2.5$ & $45.6 \pm 3.5$ & $46.5 \pm 2.4$ & $41.0 \pm 3.1$ \\
Gender (male/female) & $10 / 5$ & $9 / 6$ & $9 / 6$ & $11 / 4$ \\
PMI (h) & $22.7 \pm 2.2$ & $33.7 \pm 3.8^{a}$ & $27.5 \pm 2.8$ & $32.5 \pm 4.2^{b}$ \\
\hline
\end{tabular}

Data are reported as means \pm SEM. PMI = postmortem interval; SCZ = schizophrenia; $\mathrm{MD}=$ major depression; $\mathrm{BD}=$ bipolar depression.

Planned contrast, ${ }^{\mathrm{a}} \mathrm{P}=0.04$ and ${ }^{\mathrm{b}} \mathrm{P}=0.07$.
Labeling was seen predominantly in pyramidal neurons although some interneurons were observed interspersed between the pyramidal cells as well as in the stratum radiatum, stratum lacunosum, and molecular layers. Granular immunoreactivity was seen in the cytoplasm of nNOSIR pyramidal neurons, showing the characteristic single apical dendrite projecting perpendicular to the axis of the hippocampus, as previously described by Doyle and Slater (12). The granule cells of the dentate gyrus were weakly immunostained for nNOS. Few nNOS-IR fibers were also observed within the hippocampus.

No staining was observed when the primary antiserum was omitted from the reaction solution (Figure 2).

\section{Nissl-stained and nNOS-IR neurons}

As shown in Table 2, the numbers of Nissl-stained neurons in each sector were similar in the diagnostic groups and there were no trends of main effects of diagnosis or interactions with side $(P>0.05)$. In most sectors, the number of Nissl-stained neurons correlated significantly with the number of nNOS-IR neurons.

In general, the MD and BD groups tended to have more nNOS-IR neurons in all regions, especially in the right, and this was most statistically compelling in CA1 and subiculum while the SCZ group showed no trends of differences from controls (Figure 3 ). The detailed results are presented below in the order of the multisynaptic hippocampal pathway: entorhinal cortex-dentate gyrus-CA4-CA3-CA2CA1-subiculum.

There were no changes in cell neuron numbers in the entorhinal cortex or in CA4, except for a main effect of diagnosis after covarying for Nissl-stained neurons ( $F=$ 2.06; d.f. $=2.33 ; \mathrm{P}<0.05)$. The $\mathrm{MD}$ and $\mathrm{BD}$ groups had greater numbers of nNOS-IR neurons in relation to Nisslstained neurons than controls (planned contrasts, $P=0.03$ and $P=0.04$, respectively). In CA3 there was a diagnosis by side interaction $(P=0.04)$ in the main analysis but this was due to nNOS-IR neuron numbers in the three subjects for which there was no data in the right hemisphere in the SCZ group. This interaction was reduced in significance $(P=0.11)$ when ANOVA was repeated without the SCZ group. This trend probably reflects non-significant increases in the right hemisphere and decreases in the left hemisphere, especially in the BD group (Table 2). There were no significant group differences in neuronal number in CA2.

There were clear-cut changes in the number of nNOS-IR neurons in CA1 (Table 2, Figure 3 ). The affectively ill groups, MD and 

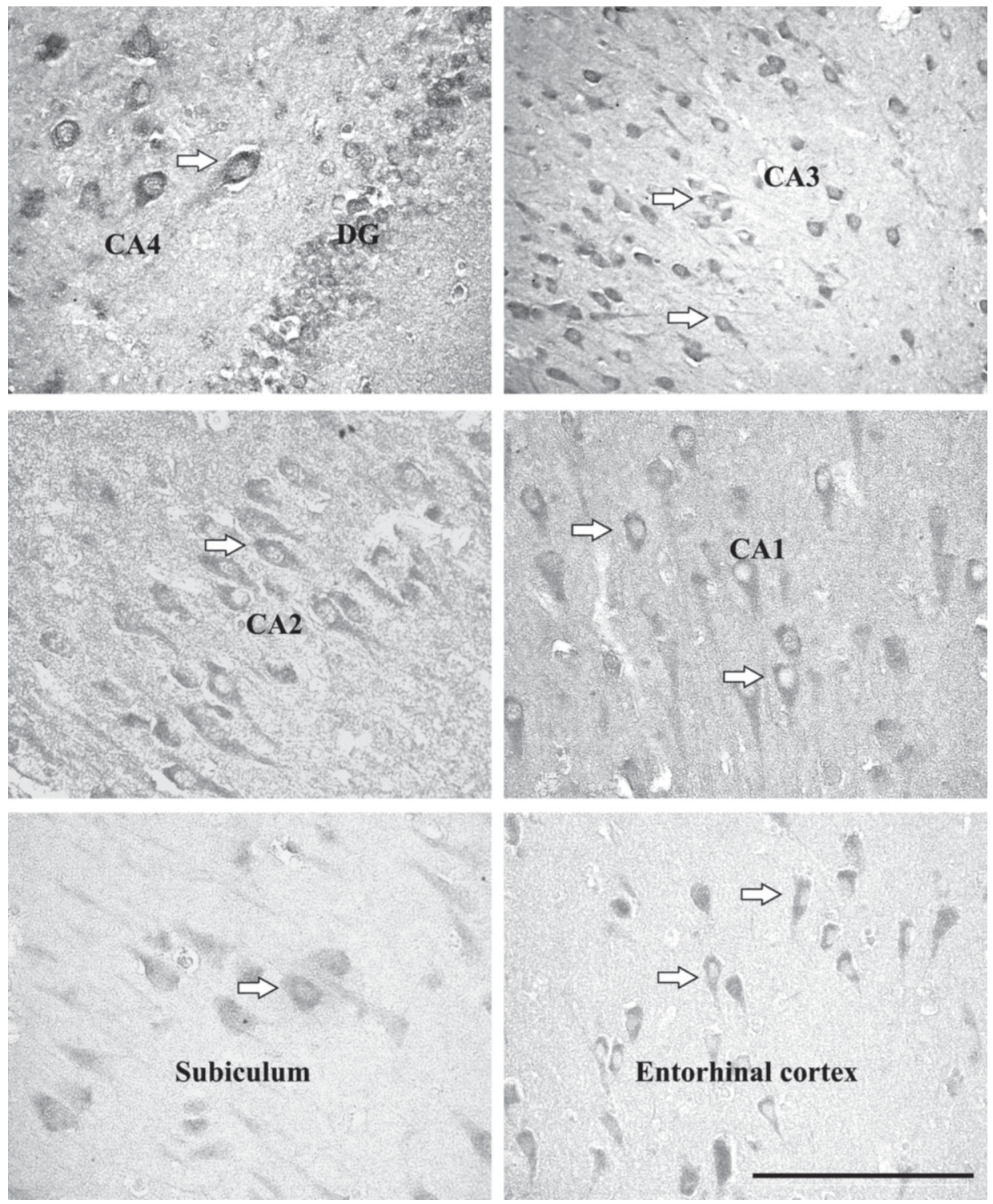

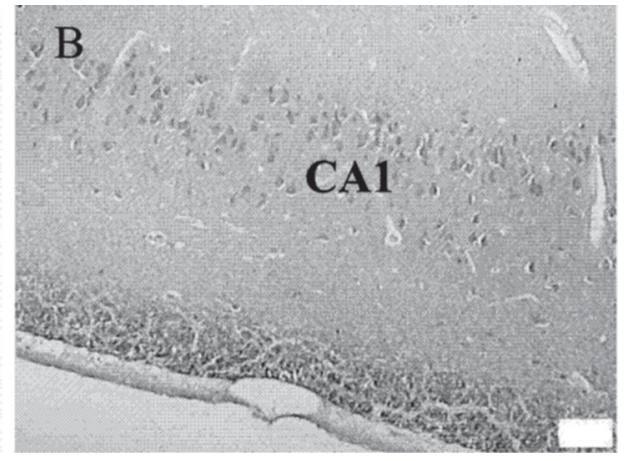

Figure 1. Neuronal nitric oxide synthase-immunoreactive (nNOS-IR) neurons in the hippocampal formation. Photomicrographs illustrating nNOS-IR neurons (10- $\mu \mathrm{m}$ sections) in the hippocampal CA4, CA3, CA2, and CA1 subfields, dentate gyrus (DG), subiculum, and entorhinal cortex of human brain. Immunohistochemistry was performed using a monoclonal anti-nNOS antibody (1:25, Transduction Laboratories). Arrows indicate nNOS-IR neurons. Bar $=100$ $\mu \mathrm{m}$ for all panels.
Figure 2. Neuronal nitric oxide synthase-immunoreactive (nNOS-IR) neurons in the hippocampal CA1 subfield. Photomicrographs illustrating a negative control (A) omitting the primary nNOS antibody from the staining protocol and nNOS-IR neurons $(B)$ in the hippocampal CA1 subfield. Arrows indicate nNOS-IR neurons. Bar $=100 \mu \mathrm{m}$ for panels $A$ and $B$. 
Table 2. Expression of neuronal nitric oxide synthase-immunoreactive (nNOS-IR) neurons in the human hippocampal formation of schizophrenia (SCZ), major depression (MD), bipolar disorder (BD), and control groups.

\begin{tabular}{|c|c|c|c|c|c|c|c|c|}
\hline \multirow[t]{2}{*}{ Area } & \multicolumn{4}{|c|}{ nNOS-IR neurons $/ 400 \mu \mathrm{m}^{2}$} & \multicolumn{4}{|c|}{ Nissl-stained neurons $/ 400 \mu \mathrm{m}^{2}$} \\
\hline & Control & SCZ & MD & $\mathrm{BD}$ & Control & SCZ & MD & $\mathrm{BD}$ \\
\hline \multicolumn{9}{|l|}{ CA4 } \\
\hline Left & $3.2 \pm 0.4$ & $3.7 \pm 0.3$ & $3.3 \pm 0.4$ & $3.2 \pm 0.4$ & $3.9 \pm 0.3$ & $3.4 \pm 0.3$ & $3.9 \pm 0.3$ & $3.4 \pm 0.2$ \\
\hline Right & $2.7 \pm 0.4$ & $3.4 \pm 0.5$ & $3.8 \pm 0.4$ & $3.6 \pm 0.4$ & $3.6 \pm 0.3$ & $3.2 \pm 0.4$ & $3.3 \pm 0.3$ & $3.4 \pm 0.3$ \\
\hline \multicolumn{9}{|l|}{ CA3 } \\
\hline Left & $9.6 \pm 0.5$ & $9.6 \pm 0.5$ & $10.0 \pm 0.5$ & $8.8 \pm 0.5$ & $10.3 \pm 0.8$ & $11.0 \pm 0.7$ & $11.6 \pm 0.7$ & $10.2 \pm 0.8$ \\
\hline Right & $9.8 \pm 0.6$ & $8.0 \pm 0.7$ & $9.7 \pm 0.6$ & $11.0 \pm 0.5$ & $10.8 \pm 0.9$ & $9.8 \pm 1.1$ & $9.6 \pm 0.8$ & $11.3 \pm 0.7$ \\
\hline \multicolumn{9}{|l|}{$\mathrm{CA} 2$} \\
\hline Left & $12.0 \pm 0.8$ & $10.5 \pm 0.7$ & $13.5 \pm 0.74$ & $12.3 \pm 1.0$ & $13.5 \pm 0.8$ & $14.7 \pm 0.7$ & $15.6 \pm 0.8$ & $13.9 \pm 0.9$ \\
\hline Right & $12.0 \pm 1.0$ & $11.1 \pm 1.2$ & $12.0 \pm 0.8$ & $11.3 \pm 0.8$ & $13.9 \pm 0.9$ & $14.6 \pm 1.3$ & $13.5 \pm 0.8$ & $14.0 \pm 0.8$ \\
\hline \multicolumn{9}{|l|}{ CA1 } \\
\hline Left & $8.1 \pm 0.5$ & $8.7 \pm 0.5$ & $8.4 \pm 0.5$ & $8.5 \pm 0.6$ & $7.7 \pm 0.7$ & $8.7 \pm 0.7$ & $8.2 \pm 0.7$ & $7.9 \pm 0.9$ \\
\hline Right & $6.6 \pm 0.5$ & $6.7 \pm 0.8$ & $9.2 \pm 0.6^{* *}$ & $8.4 \pm 0.6^{*}$ & $8.9 \pm 0.7$ & $10.4 \pm 1.0$ & $9.0 \pm 0.8$ & $9.9 \pm 0.7$ \\
\hline \multicolumn{9}{|c|}{ Subiculum } \\
\hline Left & $7.1 \pm 0.5$ & $6.2 \pm 0.4$ & $6.6 \pm 0.4$ & $6.1 \pm 0.5$ & $8.6 \pm 0.6$ & $8.2 \pm 0.5$ & $8.5 \pm 0.6$ & $8.4 \pm 0.7$ \\
\hline Right & $5.9 \pm 0.4$ & $6.7 \pm 0.6$ & $6.8 \pm 0.5$ & $6.7 \pm 0.4$ & $8.7 \pm 0.6$ & $8.7 \pm 0.7$ & $9.1 \pm 0.6$ & $7.8 \pm 0.6$ \\
\hline \multicolumn{9}{|l|}{ ECII } \\
\hline Left & $5.7 \pm 0.8$ & $5.9 \pm 0.5$ & $5.1 \pm 0.5$ & $5.5 \pm 0.6$ & $7.0 \pm 0.4$ & $7.6 \pm 0.4$ & $7.4 \pm 0.4$ & $7.3 \pm 0.5$ \\
\hline Right & $6.3 \pm 0.5$ & $5.0 \pm 0.8$ & $5.9 \pm 0.7$ & $6.8 \pm 0.5$ & $7.6 \pm 0.4$ & $7.3 \pm 0.7$ & $7.6 \pm 0.5$ & $8.1 \pm 0.4$ \\
\hline \multicolumn{9}{|l|}{ ECIII } \\
\hline Left & $6.2 \pm 0.5$ & $6.5 \pm 0.4$ & $6.6 \pm 0.5$ & $6.0 \pm 0.5$ & $6.9 \pm 0.4$ & $7.9 \pm 0.4$ & $8.2 \pm 0.4$ & $7.7 \pm 0.5$ \\
\hline Right & $5.9 \pm 0.4$ & $6.1 \pm 1.2$ & $5.1 \pm 0.7$ & $6.4 \pm 0.5$ & $7.6 \pm 0.4$ & $7.8 \pm 0.7$ & $6.8 \pm 0.5$ & $7.5 \pm 0.4$ \\
\hline
\end{tabular}

Data are reported as means \pm SEM. See text for ANOVA details.

${ }^{*} \mathrm{P}=0.05,{ }^{* *} \mathrm{P}=0.01$, planned contrasts compared to control group in the right hemisphere.

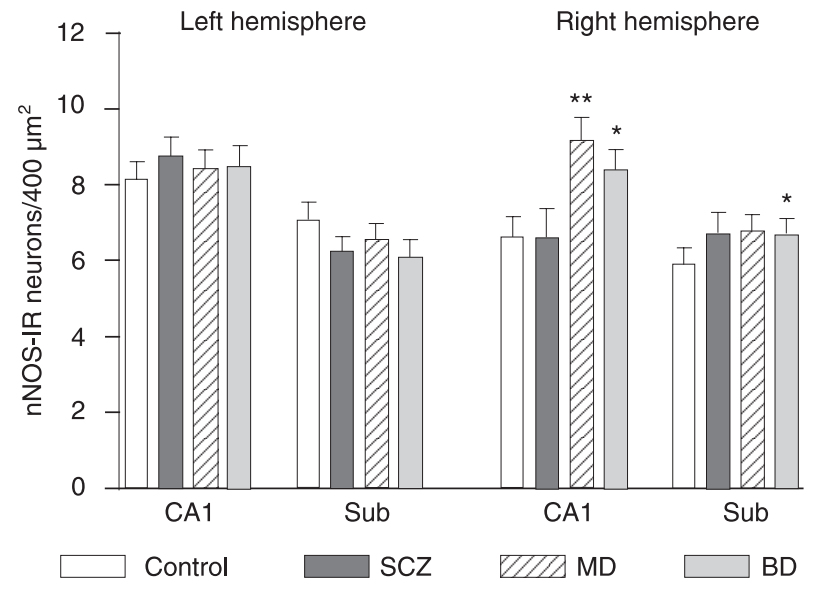

Figure 3. Expression of neuronal nitric oxide synthase-immunoreactive (nNOS-IR) neurons in the CA1 and subiculum (Sub) of hippocampal formation of schizophrenia (SCZ), major depression (MD), bipolar disorder (BD), and control groups. Observe nNOS-IR expression in subfields with significant diagnosis by side interactions. $\mathrm{N}=6-8$ subjects per group except $3-4$ subjects for $\mathrm{SCZ}$ in the right hemisphere. See text for ANOVA details. ${ }^{*} \mathrm{P}$ $=0.05,{ }^{* *} P=0.01$, planned contrasts compared to control group in the right hemisphere. 
$\mathrm{BD}$, had more nNOS-IR neurons in the right than controls, with no differences in the left region (main analysis: $F=$ 5.02 ; d.f. $=3.40 ; \mathrm{P}<0.05$, contrasts vs controls in the right: $M D, P=0.003 ; B D, P=0.03)$. These effects were significant in all analyses. This pattern was repeated in the subiculum of BD and was statistically significant in two subsidiary ANOVA tests $(P<0.05)$.

\section{Clinical correlates}

There were no group differences between affectively ill subjects (i.e., MD combined with BD) on or off antidepressants ( $N=17$ vs 18 , respectively), benzodiazepines $(N=7$ vs 19), or lithium ( $N=6$ vs 20), except in CA3 where lithiumtreated subjects had greater numbers of nNOS-IR neurons than non-lithium-treated subjects (mean $\pm \mathrm{SEM} / \mathrm{N}$ : lithiumtreated, $10.9 \pm 0.48 / 5$; non-treated, $9.5 \pm 0.28 / 18 ; \mathrm{t}=2.29$; $P=0.03)$.

There were no differences when comparing deaths by suicide to death by natural causes within or between the diagnostic groups.

Duration of illness showed no relationship with nNOS$\mathrm{IR}$ counts in the combined MD and BD groups or in the BD group alone or in subjects on or off antidepressants.

\section{Discussion}

The present study provides evidence that nNOS expression is increased in CA1 and subiculum of patients with affective disorders. This increase was significantly more marked in samples from the right hemisphere and was specific to the MD and BD groups, with no main effect involving the SCZ group. While there were too few observations in the SCZ group to completely rule out rightlateralized changes, there was no trend to an increase in nNOS-IR neurons in CA1.

The pattern of nNOS expression observed in the hippocampal formation and entorhinal cortex in the present study agrees with other reports $(11,12)$. Although no quantitative description about the density of nNOS-IR neurons in the hippocampus has been previously reported, there appears to be a greater number of nNOS-IR neurons in the present study than reported by others. The mouse monoclonal anti-nNOS antibody used has been shown to react specifically with the 150-kDa human nNOS of the recombinant protein or brain extract (Western blot), showing sufficient isoform specificity to undertake reliable immunohistochemical staining (30). We may have observed a greater density because previous studies used different fixation techniques (formalin-fixed tissue versus frozen sections) and detected nNOS expression using polyclonal antibodies or NADPH-diaphorase histochemistry.
We examined the possible influence of confounding variables. Only time in formalin differed markedly between groups and PMIs tended to show group differences (Table 1). However, no variable correlated with nNOS counts, so group differences are unlikely to have contributed to group differences in cell counts. Furthermore, these variables did not differ between the SCZ group and the affectively ill groups (MD and BD) while changes in nNOS counts were limited to the affective groups.

The changes observed were more marked in the right region and this may reflect the specialization of the right hemisphere for affective processing in humans. Several anatomical and functional changes in clinical studies of MD are lateralized (31). O'Brien et al. (32) reported right hippocampal atrophy in elderly patients with depression. A recent meta-analysis found that right, but not left, hippocampal atrophy was associated with number of previous episodes of depression (1). Furthermore, the right hemisphere has been implicated in the pathogenesis of affective instability by studies on stroke and temporal lobe epilepsy $(33,34)$.

Stockmeier et al. (4) reported increased packing of pyramidal neurons and glia in postmortem hippocampus from the right hemispheres of patients with MD. They concluded that loss of neuropil may occur in MD which may contribute to the volume reduction noted in imaging studies. These investigators also observed a reduced soma size in the hippocampal formation in depression, as did Chana et al. (35) in some laminae of the cingulate cortex. Increased packing due to loss of neuropil could explain our finding of increased nNOS counts but only if loss of neuropil was right-sided. We cannot exclude this possibility from our study since we did not perform morphometric analysis of the neurons. However, this explanation for increased nNOS cell counts would also require the assumption of a right lateralized change in cell size and, in addition, that non-nNOS and nNOS-IR neurons show differential reduction in size. Another possible confounder is that differential shrinkage of the sections may have occurred during histological processing in our affectively ill groups compared to the control and SCZ groups, as reported by Stockmeier et al. (4). However, this seems to be an unlikely confounder in the present study since cells were counted throughout the thickness of the sampling frame in all groups.

There was no evidence that increased nNOS-IR neurons in the affective groups were attenuated in subjects treated with antidepressants. However, the fact that approximately half the patients had died by suicide and that others were symptomatic suggests that many patients had not responded to treatment. Indeed, the lack of effect of drugs on nNOS counts could indicate a failure to suppress 
hippocampal function, as seen in a PET study of fluoxetine non-responders (20). This assumption is supported by the fact that imipramine withdrawal was associated with a significant increase in NOS activity in the hippocampus of rats (23).

Increased numbers of nNOS-IR neurons in CA1 of MD and BD seem to contradict the prediction that high circulating glucocorticoid concentrations in affective disorders might have neurotoxic effects through NMDA-mediated nNOS expression in the hippocampus (8). It is possible, however, that nNOS-positive neurons are resistant to cytotoxicity and so become more densely packed as other neurons die. Resistance of nNOS neurons has been reported in Alzheimer's disease (36), Huntington's disease (37) and NMDA-mediated neurotoxicity itself (8). Nevertheless, the asymmetric changes provide a strong argument against increased packing as the mechanism of increased nNOS-IR neurons; it is difficult to envisage a unilateral neurotoxic effect of stress. It is very likely that the hippocampi from the MD and BD groups had undergone prolonged exposure to glucocorticoids, especially because many were from suicide victims. Indeed, it has been reported that glucocorticoid receptor mRNA expression is decreased in the postmortem hippocampus but this occurred in all diagnostic groups including SCZ, albeit in different subregions (38). However, nNOS counts in any hippocampal subfield did not show any tendency to decrease. We conclude that our results are not likely to be due to cytotoxic effects of elevated glucocorticoid exposure. However, it remains possible that increased nNOS expression is initiated by glucocorticoids.

In conclusion, we provide evidence for an increased number of neurons expressing nNOS-IR in the hippocampus of patients with MD and BD. These results are compatible with our main prediction that hippocampi from affectively ill patients would show increased nNOS expression and reinforce current interest in the NMDA-NO pathway as a target for the pathophysiology of affective disorders and antidepressant drug development.

\section{Acknowledgments}

Postmortem brains were donated by the Stanley Foundation Brain Collection, Stanley Foundation, Muscatine, IA, USA, courtesy of Drs. Michael B. Knable, E. Fuller Torrey, Maree J. Webster, and Robert H. Yolken.

\section{References}

1. Campbell $\mathrm{S}$, Macqueen $\mathrm{G}$. The role of the hippocampus in the pathophysiology of major depression. J Psychiatry Neurosci 2004; 29: 417-426.

2. Sheline YI, Wang PW, Gado MH, Csernansky JG, Vannier MW. Hippocampal atrophy in recurrent major depression. Proc Natl Acad Sci U S A 1996; 93: 3908-3913.

3. Bremner JD, Narayan M, Anderson ER, Staib LH, Miller HL, Charney DS. Hippocampal volume reduction in major depression. Am J Psychiatry 2000; 157: 115-118.

4. Stockmeier CA, Mahajan GJ, Konick LC, Overholser JC, Jurjus GJ, Meltzer HY, et al. Cellular changes in the postmortem hippocampus in major depression. Biol Psychiatry 2004; 56: 640-650.

5. Uno H, Tarara R, Else JG, Suleman MA, Sapolsky RM. Hippocampal damage associated with prolonged and fatal stress in primates. J Neurosci 1989; 9: 1705-1711.

6. McEwen BS, Magarinos AM, Reagan LP. Structural plasticity and tianeptine: cellular and molecular targets. Eur Psychiatry 2002; 17 (Suppl 3): 318-330.

7. Garthwaite J. Glutamate, nitric oxide and cell-cell signalling in the nervous system. Trends Neurosci 1991; 14: 60-67.

8. Dawson VL, Dawson TM. Nitric oxide in neurodegeneration. Prog Brain Res 1998; 118: 215-229.

9. Yanik M, Vural H, Tutkun H, Zoroglu SS, Savas HA, Herken $\mathrm{H}$, et al. The role of the arginine-nitric oxide pathway in the pathogenesis of bipolar affective disorder. Eur Arch Psychiatry Clin Neurosci 2004; 254: 43-47.
10. Law AJ, Weickert CS, Webster MJ, Herman MM, Kleinman JE, Harrison PJ. Expression of NMDA receptor NR1, NR2A and NR2B subunit mRNAs during development of the human hippocampal formation. Eur J Neurosci 2003; 18: 11971205.

11. Egberongbe YI, Gentleman SM, Falkai P, Bogerts B, Polak $\mathrm{JM}$, Roberts GW. The distribution of nitric oxide synthase immunoreactivity in the human brain. Neuroscience 1994; 59: 561-578.

12. Doyle CA, Slater P. Localization of neuronal and endothelial nitric oxide synthase isoforms in human hippocampus. Neuroscience 1997; 76: 387-395.

13. Harkin A, Connor TJ, Walsh M, St John N, Kelly JP. Serotonergic mediation of the antidepressant-like effects of nitric oxide synthase inhibitors. Neuropharmacology 2003; 44: 616-623.

14. Paul IA, Skolnick P. Glutamate and depression: clinical and preclinical studies. Ann N Y Acad Sci 2003; 1003: 250-272.

15. Joca SR, Guimarães FS. Inhibition of neuronal nitric oxide synthase in the rat hippocampus induces antidepressantlike effects. Psychopharmacology 2006; 185: 298-305.

16. Karolewicz B, Bruce KH, Lee B, Paul IA. Nitric oxide synthase inhibitors have antidepressant-like properties in mice. 2. Chronic treatment results in downregulation of cortical beta-adrenoceptors. Eur J Pharmacol 1999; 372: 215-220.

17. Zarate CA Jr, Singh JB, Carlson PJ, Brutsche NE, Ameli R, Luckenbaugh DA, et al. A randomized trial of an $\mathrm{N}$-methyl- 
D-aspartate antagonist in treatment-resistant major depression. Arch Gen Psychiatry 2006; 63: 856-864.

18. Naylor GJ, Smith AH, Connelly P. A controlled trial of methylene blue in severe depressive illness. Biol Psychiatry 1987; 22: 657-659.

19. Suzuki E, Yagi G, Nakaki T, Kanba S, Asai M. Elevated plasma nitrate levels in depressive states. J Affect Disord 2001; 63: 221-224.

20. Mayberg HS, Brannan SK, Tekell JL, Silva JA, Mahurin RK, McGinnis S, et al. Regional metabolic effects of fluoxetine in major depression: serial changes and relationship to clinical response. Biol Psychiatry 2000; 48: 830-843.

21. Bonvento G, Sibson N, Pellerin L. Does glutamate image your thoughts? Trends Neurosci 2002; 25: 359-364.

22. Li YF, Zhang YZ, Liu YQ, Wang HL, Cao JB, Guan TT, et al. Inhibition of N-methyl-D-aspartate receptor function appears to be one of the common actions for antidepressants. $J$ Psychopharmacol 2006; 20: 629-635.

23. Deakin JFW, Graeff FG. 5-HT and mechanisms of defense. J Psychopharmacol 1991; 5: 305-315.

24. Harvey BH, Retief R, Korff A, Wegener G. Increased hippocampal nitric oxide synthase activity and stress responsiveness after imipramine discontinuation: role of $5 \mathrm{HT} 2 \mathrm{~A} / \mathrm{C}$ receptors. Metab Brain Dis 2006; 21: 211-220.

25. Torrey EF, Webster M, Knable M, Johnston N, Yolken RH. The Stanley Foundation Brain Collection and Neuropathology Consortium. Schizophr Res 2000; 44: 151-155.

26. Benes FM, Lange N. Two-dimensional versus three-dimensional cell counting: a practical perspective. Trends Neurosci 2001; 24: 11-17.

27. Thorns V, Hansen L, Masliah E. nNOS expressing neurons in the entorhinal cortex and hippocampus are affected in patients with Alzheimer's disease. Exp Neurol 1998; 150: 14-20.

28. Katsuse O, Iseki E, Kosaka K. Immunohistochemical study of the expression of cytokines and nitric oxide synthases in brains of patients with dementia with Lewy bodies. Neuropathology 2003; 23: 9-15.

29. Paxinos G, Mai JK. The human nervous system. San Diego: Academic Press; 2003.

30. Coers W, Timens W, Kempinga C, Klok PA, Moshage H. Specificity of antibodies to nitric oxide synthase isoforms in human, guinea pig, rat, and mouse tissues. J Histochem Cytochem 1998; 46: 1385-1392.

31. Drevets WC. Neuroimaging studies of mood disorders. Biol Psychiatry 2000; 48: 813-829.

32. O'Brien JT, Lloyd A, McKeith I, Gholkar A, Ferrier N. A longitudinal study of hippocampal volume, cortisol levels, and cognition in older depressed subjects. Am J Psychiatry 2004; 161: 2081-2090.

33. Ghika-Schmid F, Bogousslavsky J. Affective disorders following stroke. Eur Neurol 1997; 38: 75-81.

34. MacHale SM, O'Rourke SJ, Wardlaw JM, Dennis MS. Depression and its relation to lesion location after stroke. $J$ Neurol Neurosurg Psychiatry 1998; 64: 371-374.

35. Chana G, Landau S, Beasley C, Everall IP, Cotter D. Twodimensional assessment of cytoarchitecture in the anterior cingulate cortex in major depressive disorder, bipolar disorder, and schizophrenia: evidence for decreased neuronal somal size and increased neuronal density. Biol Psychiatry 2003; 53: 1086-1098.

36. Tao Z, Van GD, Lammens M, Dom R. NADPH-diaphorasecontaining neurons in cortex, subcortical white matter and neostriatum are selectively spared in Alzheimer's disease. Dement Geriatr Cogn Disord 1999; 10: 460-468.

37. Ferrante RJ, Kowall NW, Beal MF, Richardson EP Jr, Bird ED, Martin JB. Selective sparing of a class of striatal neurons in Huntington's disease. Science 1985; 230: 561-563.

38. Webster MJ, Knable MB, O'Grady J, Orthmann J, Weickert CS. Regional specificity of brain glucocorticoid receptor mRNA alterations in subjects with schizophrenia and mood disorders. Mol Psychiatry 2002; 7: 985-994, 924. 